\title{
An Improved Method for the Processing of Signals Contaminated with Strong Common-Mode Periodic Noise in Correlation Velocity Measurement
}

\author{
Kamel Reda* and Yong Yan** \\ School of Engineering and Digital Arts, University of Kent, Canterbury CT2 7NT, U.K. \\ ${ }^{*}$ Member, IEEE; ** Fellow, IEEE
}

\begin{abstract}
Electrostatic sensors have been successfully used for the velocity measurement of pneumatically conveyed particles and the rotational speed measurement. However, the signal from an electrostatic sensor is usually vulnerable and susceptible to contamination in a hostile environment. The acquired original signal may be contaminated by different types of noise that can be within or outside the frequency range of the signal. This paper presents a novel correlation signal processing method to minimise the impact of noise in the signal through a de-noising process and hence improve the performance of correlation-based measurements in general. The method is applied to the rotational speed measurement based on electrostatic sensors in particular. The de-noising process is an essential task in digital signal processing to improve the signal-to-noise ratio before implementing the measurement algorithm. A hybrid de-noising method is proposed to combine a cut-off frequency method to remove the noise components outside the signal bandwidth and a median filter to smooth the signal. Subsequently, the signal is de-noised in the time domain by employing an advanced digital filtering method based on correlation techniques to suppress the noise frequency components mixed with the original signal. The rotational speed measurement system with the proposed technique has proven to be effective in de-noising signals that are buried in noise with which they are correlated. Moreover, the technique is capable of producing more accurate and repeatable measurements with a wider measurement range than the existing system. Experimental results suggest that the relative error of the improved system is mostly within $\pm 0.1 \%$ over the speed range of $300 \mathrm{rpm}-3000 \mathrm{rpm}$ and within $\pm 0.2 \%$ over the speed range of $40-300 \mathrm{rpm}$.
\end{abstract}

Index Terms- Buried signal, correlation measurement, de-noising, electrostatic sensors, rotational speed.

\section{INTRODUCTION}

Correlation techniques have been applied extensively and successfully in multiphase flow measurement over the past two decades [1]-[3]. In recent years extensive research and experimental investigations have been conducted on the use of electrostatic sensors in conjunction with correlation signal processing techniques for rotational speed measurement [4]-[6]. Experimental results have shown a maximum error of no greater than $\pm 1.5 \%$ over the speed range from $500 \mathrm{rpm}$ to $3000 \mathrm{rpm}$. Much more recently, the method has been developed further by incorporating a data fusion algorithm, capable of producing more accurate measurements with a maximum error within $\pm 0.5 \%$ over the range of $200 \mathrm{rpm}$ to $3000 \mathrm{rpm}$ [7]. Although the proposed electrostatic sensing technique has successfully achieved a rotational measurement for rotary shafts, it has some limitations that will be dealt with in this paper. The existing electrostatic sensors depend primarily on the rotational speed, material properties and surface conditions of the shaft [4]. Consequently, the speed measurement is susceptible to environmental conditions, among which humidity, vibration and electromagnetic interferences are considered to be the main factors. In a hostile environment and at low rotational speed $(<200 \mathrm{rpm})$, the signal-to- noise ratio may deteriorate as the induced electrostatic charge on the electrode decreases with the discharge effect and in the presence of strong noises, making the system ineffective for low rotational speed measurement. The present study is based on the previous work and concentrates on the improvement of the performance of the measurement system. The proposed system aims at providing more accurate and repeatable measurements over a wider range of rotational speeds. To tackle the aforementioned challenge, additional signal processing strategies are implemented to improve the performance of the measurement system. The proposed technique uses dual electrostatic sensors coupled with an improved signal processing algorithm to enhance the signal-to-noise ratio and to widen the measurement range. The basic principle and the detailed structure of the rotational speed measurement system based on dual electrostatic sensors have been reported previously [5]. The rotational speed is derived from the period $(T)$ of the sensor output signals or from the time delay $(\tau)$ between them. Moreover, both $(T)$ and $(\tau)$ can be determined from the autocorrelation and cross-correlation of the output signals, respectively. A series of experiments was conducted on purpose-built test rigs to evaluate the performance of the improved system in terms of accuracy, repeatability, rangeability and robustness. 


\section{SIGNAL ANALYSIS AND DE-NOISING APPROACH}

\section{A. Signal Analysis}

The output signal from an electrostatic sensor is a random and low pass signal as shown in Fig. 1. The signal is also periodic due to the rotational motion. Its bandwidth depends on the geometric properties of the electrode and size of the rotor [8]. The bandwidth is proportional to the rotational speed [8]. Fig. 1 shows a typical noisefree signal and the corresponding spectrum.

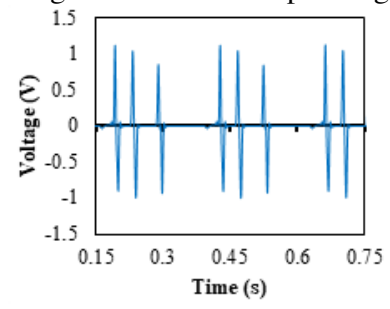

(a)

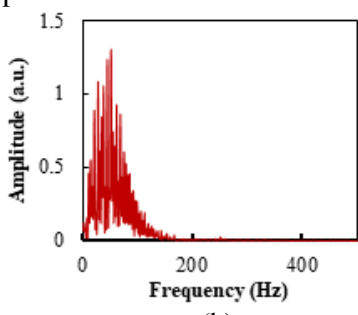

(b)
Fig. 1. Typical example of a noise-free signal and corresponding frequency spectrum at $200 \mathrm{rpm}$. (a) Noise-free signal. (b) Power spectrum of signal.

The signal from the electrostatic sensor is usually weak. Thus, various extrinsic and intrinsic noises can be superimposed on it, resulting in a contaminated signal at the output of the conditioning circuit. In some extremely poor conditions, the signal can be partly or totally buried in a strong noise (Fig. 2). This additive noise significantly degrades the quality of the signal, which can be crucial for signal processing, as a contaminated signal leads to erroneous measurement results.

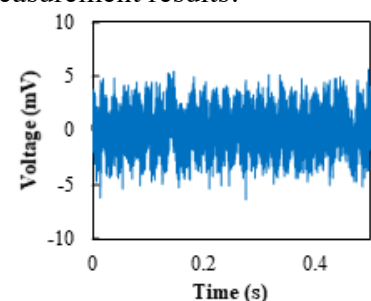

(a)

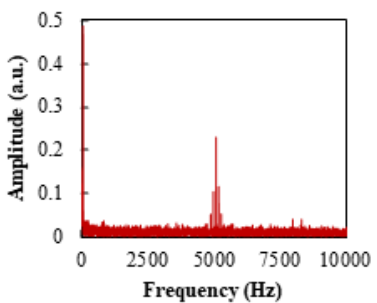

(b)
Fig. 2. Typical example of a noisy signal and corresponding frequency spectrum. (a) Noisy signal. (b) Power spectrum of the noisy signal.

The types of the source noise that are mixed with the original signal need to be considered when selecting de-noising methods. Generally, the acquired signal is contaminated by different types of noise that can be within or outside the frequency range of the original signal. In some extremely poor conditions, the signal is correlated with noises in which it is buried. The most common sources of noise are the power line interference, vibration of the mechanical system and intrinsic random noise. Power line interference originates primarily from the electromagnetic field of the power lines and the improper grounding of the signal conditioning circuit. The power line noise is centered at $50 \mathrm{~Hz}$ or $60 \mathrm{~Hz}$ with a bandwidth no more than $1 \mathrm{~Hz}$ and is mostly rejected by the earthed metal screen of the sensor $[9,10]$. However, adding a screen to cover the electrode and signal conditioning circuit cannot completely reject the external electrical interference. Furthermore, a traditional notch filter cannot be used to remove the residual noise since its frequency component $(50 \mathrm{~Hz}$ or $60 \mathrm{~Hz})$ may be within the spectrum of the original signal. Thus, a more advanced digital filtering method needs to be employed to suppress this residual noise. The second type of noise that may contaminate the signal is due to the vibration of the mechanical system. Although the adopted method of signal acquisition (current-to-voltage convertor) does not require consideration of the stray capacitance of the electrode in the acquisition circuit, it is still sensitive to the vibration of the sensor and the connecting cable between the sensor and the data acquisition unit. Even tiny displacements of charge in connecting cables due to physical handling can produce current large enough to cause significant noise in the system. The interference frequency of this type of noise is usually composed of the main rotational frequency and its harmonics in addition to high frequency components due to vibration of the connecting cables. Finally, intrinsic noise can be superimposed on the original signal from the signal conditioning circuit. This noise is approximated to white noise which has a continuous frequency spectrum distribution. For that reason, the signal conditioning circuit was designed with extremely low noise using high-performance components [11]. Additionally, a low-pass filter was added to the preprocessing to eliminate the high-frequency noise.

\section{B. Filtering of high-frequency noise}

Although the signal conditioner comprises a low-pass filter that helps to remove high-frequency noise, nevertheless, other types of noise, cannot be suppressed by the analogue circuit. To address this problem, a digital filtering algorithm is proposed which combines a cut-off frequency method based on the Fourier transform and a median filter, to remove the high-frequency noise components and also to smooth the signal. A $5^{\text {th }}$-order IIR Butterworth filter is employed to suppress noise outside the signal bandwidth. Then, a median filter with a window size of 50 is applied to smooth the signal. Figs. 3 and 4 show a typical example of the filtered signals $S_{I n f}$ and $S_{2 n f}$ and the corresponding power spectra, respectively. The periodicity of the signals, due to the continuous motion of the shaft, becomes apparent after applying the low-pass filter.

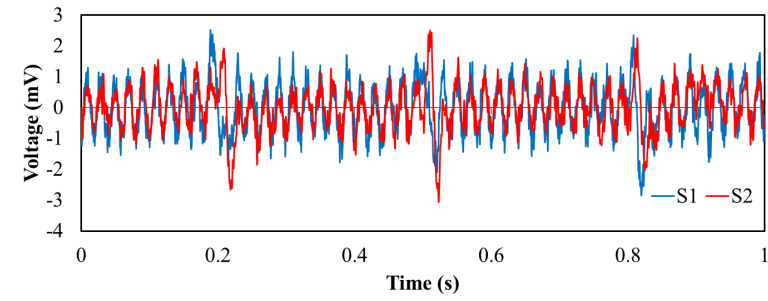

Fig. 3. Filtered signals $S_{1 n f}$ and $S_{2 n f}$ from a dual electrostatic sensor.

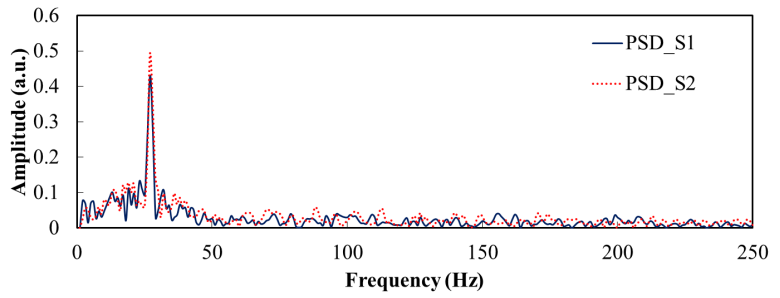

Fig. 4. Spectra of the filtered signals in Fig. 3.

Figs. 5 and 6 illustrate the autocorrelation function of the filtered signal $S_{I n f}$ and the cross-correlation function of the two filtered signals $S_{1 n f}$ and $S_{2 n f}$. The two correlation functions show that the filtered signals are still buried in strong noise since the dominant peaks, from which the periodicity of the signals is determined, are not clearly defined and stable. Although high-frequency noise has been eliminated using digital low-pass filters, the filtered signals are still contaminated with noises that may be correlated with the original 
signals and their spectra are within the frequency band of the original signals. Thus, a simple cut-off frequency method is not sufficient to remove such types of noise, making the measurement invalid. Therefore, the filtered signals should be further de-noised using an advanced digital filtering method to remove the frequency components mixed with the desired signal.

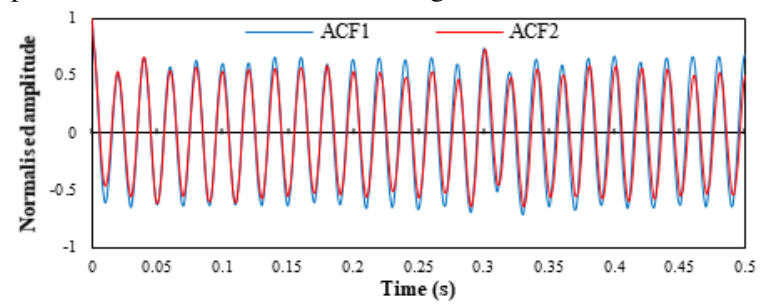

Fig. 5. Autocorrelation functions of the filtered signal $S_{1 n f}$ and $S_{2 n f \text {. }}$

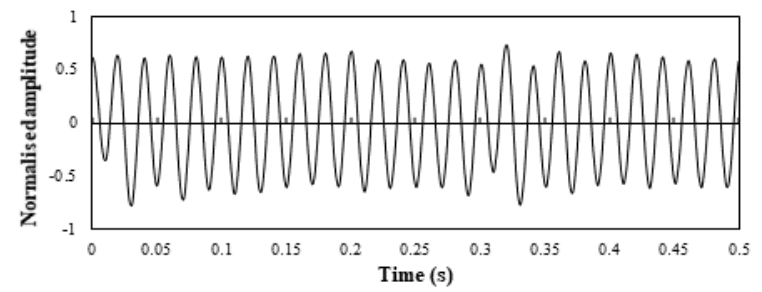

Fig. 6. Cross-correlation function of the two filtered signals $S_{1 n f}$ and $S_{2 n f}$.

\section{Filtering of common mode noise}

The proposed sensing system for rotational speed measurement is based on a dual electrostatic sensor, comprising two electrodes and connected to two independent conditioning circuits [5]. Consequently, the dual sensor derives two signals $S_{I}(t)$ and $S_{2}(t)$, similar to each other apart from the time delay between them (Fig. 3). Since the electrodes and conditioning circuits of the two sensors are almost identical, it can be assumed that they are exposed to common mode noises which they may be correlated with, and their spectra are within the spectrum of the signal. Noises due to power line interference and vibration of the rig can be considered as common mode noise. Thus, employing a method to reduce the common mode noise would suppress most of these noises.

The autocorrelation function $\left(R_{S I S I}\right)$ of the noise-free signal $S_{I}(t)$ and the cross-correlation function $\left(R_{S I S 2}\right)$ of the noise-free signals, $S_{l}(t)$ and $S_{2}(t)$, can be written as

$$
\begin{aligned}
& R_{S_{1} S_{1}}(m)=\sum_{k=1}^{N} S_{1}(k) S_{1}(k+m) \\
& R_{S_{1} S_{2}}(m)=\sum_{k=1}^{N} S_{1}(k) S_{2}(k+m)
\end{aligned}
$$

where $\mathrm{N}$ is the number of samples in the correlation computation and $\mathrm{m}(\mathrm{m}=0, \ldots, \mathrm{N})$ is the number of delayed points.

If, however, the two signals $\left(S_{l}(t)\right)$ and $\left(S_{2}(t)\right)$ are contaminated with a strong common mode periodic noise $n(t)$ with which they are correlated, the autocorrelation function $\left(R_{S I n S I n}\right)$ of the noisy signal $\left(S_{I n}(t)\right)$ and the cross-correlation function $\left(R_{S I n S 2 n}\right)$ of the noisy signals, $S_{1 n}(t)$ and $S_{2 n}(t)$, become

$$
\begin{gathered}
\boldsymbol{R}_{S_{1 n} S_{1 n}}(\boldsymbol{m})=\sum_{k=1}^{N} S_{1 n}(\boldsymbol{k}) S_{1 n}(\boldsymbol{k}+\boldsymbol{m}) \\
R_{S_{1 n} S_{1 n}}(m)=\sum_{k=1}^{N}\left[\left(S_{1}(k)+n(k)\right)\left(S_{1}(k+m)+n(k+m)\right)\right] \\
R_{S_{1 n} S_{1 n}}(m)=\sum_{k=1}^{N} S_{1}(k) S_{1}(k+m)+\sum_{k=1}^{N} S_{1}(k) n(k+m)
\end{gathered}
$$




\section{EXPERIMENTAL VALIDATION}

Experimental tests were conducted on two purpose-built test rigs [11] as shown in Fig. 8 to validate the performance of the improved measurement system against an optical tachometer as a reference meter. The sensor output signals are sampled at a frequency of $50 \mathrm{kHz}$ using a high-performance data acquisition unit (NI USB-6351) with 16-bit ADC and processed on a host computer through an optimized code using LabView [5, 11]. The speed measurement using the proposed method and conventional method are plotted in Fig. 9. Each measured speed is the average of 20 instantaneous measurements.

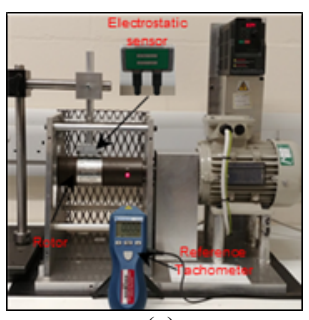

(a)

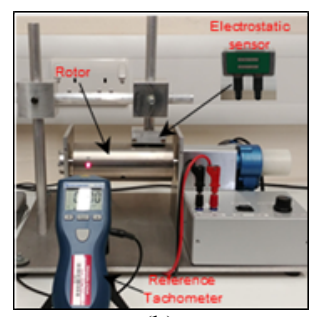

(b)
Fig. 8. Test Rig. (a) Test rig for 300-3000 rpm. (b) Test rig for 0-300 rpm.

It can be seen that the two methods can achieve the rotational speed measurements over the range between $500 \mathrm{rpm}$ and $3000 \mathrm{rpm}$. However, the proposed method outperforms the conventional one in terms of accuracy as shown in Fig. 10. The relative error using the modified correlation method is within $\pm 0.1 \%$ and with a repeatability of $<0.05 \%$, compared to $<0.33 \%$ in the data fusion method [7].

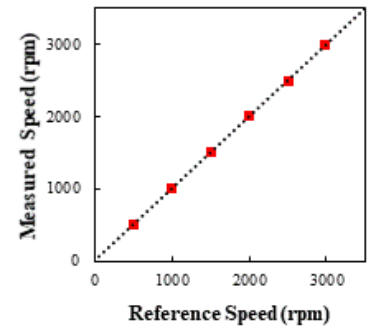

(a)

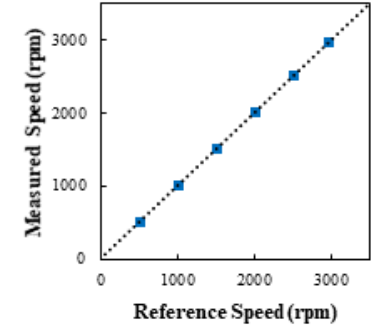

(b)
Fig. 9. Comparison of measured speeds up to $3000 \mathrm{rpm}$. (a) Conventional correlation method. (b) Modified correlation method.

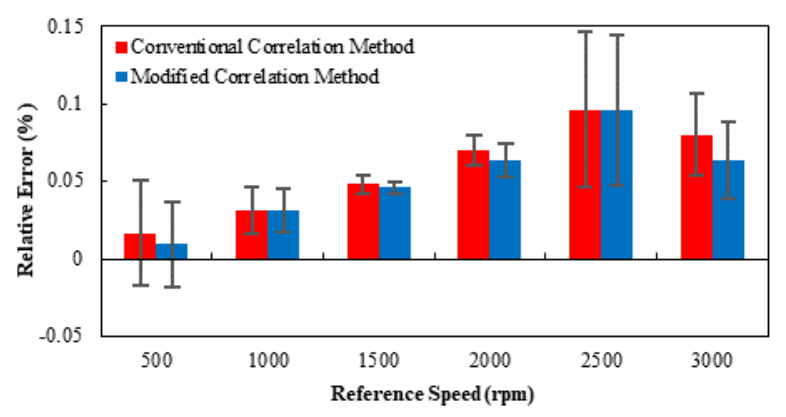

Fig. 10. Relative error of the measured rotational speed.

A second series of experiments was conducted for the speed range from $40 \mathrm{rpm}$ to $300 \mathrm{rpm}$. Fig. 11 shows that the lower end range of the conventional method is $160 \mathrm{rpm}$ whereas the proposed method can achieve valid measurements down to $40 \mathrm{rpm}$ with a relative error within $\pm 0.2 \%$ and a maximum repeatability of $0.7 \%$ (Fig. 12).

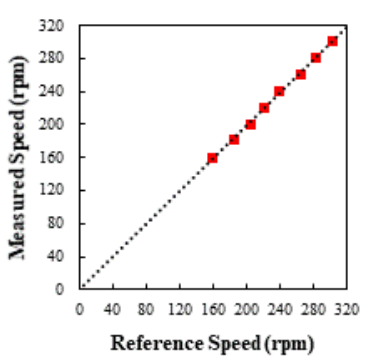

(a)

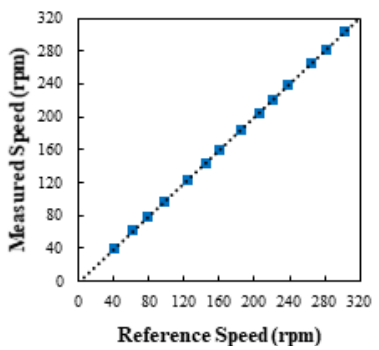

(b)
Fig. 11. Comparison of measured speeds up to $300 \mathrm{rpm}$. (a) Conventional correlation method. (b) Modified correlation method.

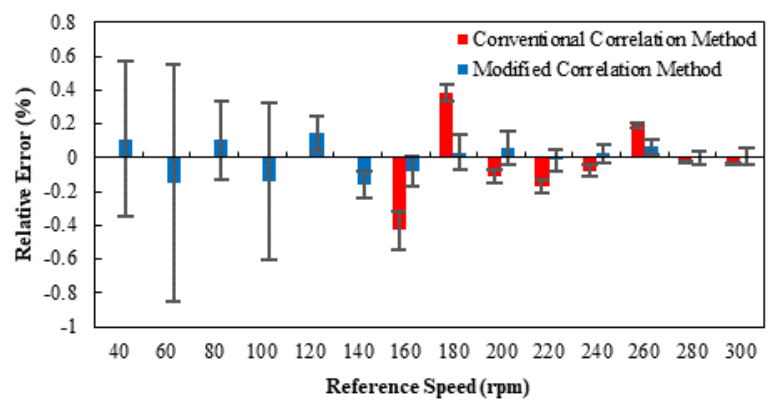

Fig. 12. Relative error of the measured speed for low speed range.

\section{CONCLUSION}

A de-noising method for signals used in correlation measurement systems has been proposed and evaluated. The improved correlation technique has been shown to improve the performance and enhance the robustness of the rotational speed measurement system. The modified correlation method has proven to be effective in a replicated hostile environment where the original signals are contaminated with correlated strong common-mode periodic noises. This technique is suitable for any correlation-based measurement where there is strong periodic noise. The lab-scale experimental results have demonstrated that the measurement error is within $\pm 0.2 \%$ over the speed range from $40 \mathrm{rpm}$ to $3000 \mathrm{rpm}$ with a repeatability of $<0.7 \%$. Additionally, the lower end of the measurement range has been extended from $160 \mathrm{rpm}$ to $40 \mathrm{rpm}$. The advantages of this technique over the conventional method are more demonstrable when the signal-to-noise ratio is very low where the peak in the conventional correlation function is not apparent and stable. Field trials should be conducted in the near future to assess the effectiveness of the improved system under practical conditions.

\section{REFERENCES}

[1] Y. Liu et al, "An adaptive algorithm for cross-correlation velocity measurement," in 8th IEEE Int. Symp. on Instrum. Control Tech., London, UK, July 2012, pp. 96-100.

[2] J. Gajewski, "Accuracy of cross correlation velocity measurements in two-phase gassolid flows," Flow Measurement and Instrumentation, vol. 30, pp. 133-137, 2013.

[3] Z. Cui et al, "Flow velocity measurement by cross-correlation with tailored modulation," in IEEE International Instrumentation and Measurement Technology Conference (I2MTC) Proceedings, May 2015, pp. 1906 - 1909.

[4] L. Wang et al, "Rotational speed measurement through electrostatic sensing and correlation signal processing," IEEE Trans. Instrum.Meas, vol. 63, pp. 1190-1199, 2014. [5] L. Wang et al, "Rotational speed measurement using single and dual electrostatic sensors," IEEE Sensors Journal, vol. 15, (3), pp. 1784-1793, 2015.

[6] L. Li et al, "Use of double correlation techniques for the improvement of rotation speed measurement based on electrostatic sensors," Meas. Sci. Technol., vol. 27, pp. 025004, 2016. 
[7] L. Wang, Y. Yan and K. Reda, "Enhancing the performance of a rotational speed measurement system through data fusion," in IOP Journal of Physics: Conf. Series (IMEKO 2018), Belfast, UK, Sept. 2018, vol. 1065, Issue 7, pp. 1-1.

[8] L. Wang and Y. Yan, "Mathematical modelling and experimental validation of electrostatic sensors for rotational speed measurement," Meas. Sci. Technol., vol. 25, (11), pp. 115101, 2014

[9] J. Gajewski, "Non-intrusive solids charge and mass flow measurements with an electrostatic flow probe," Journal of Electrostatics, vol. 46, (4), pp. 271-284, 1999.

[10] C. Xu et al, "Sensing characteristics of electrostatic inductive sensor for flow parameters measurement of pneumatically conveyed particles," Journal of Electrostatics, vol. 65, (9), pp.582-592, 2007.

[11] K. Reda and Y. Yan, "Vibration measurement of an unbalanced metallic shaft using electrostatic sensors," IEEE Trans. Instrum.Meas, vol. 68, (5), pp.1467-1476, 2019. 